\title{
A Quantum Leap in Diagnostic and Analytical Science: Next Generation Sensing Devices Targeted on Distant Cell-To-Cell Communication
}

Spyridon Kintzios*

Department of Agricultural Biotechnology, Agricultural University of Athens, Greece

\section{Editorial}

Advances in the field of diagnostic science have allowed the development of analytical tools able to detect target molecules at trace quantities. At the front of biosensor research, designers of commercial or semi-commercial systems steadily try to satisfy as many as possible operational requirements within a single biorecognition platform, for example high assay speed, reproducibility, accuracy, selectivity and sensitivity, as well as extended sensor storability under normal conditions. In addition, the cost of each assay must be kept minimum, along with a minimal sample volume and minimal sample preparation time. In order to have a truly portable, field-applicable system, the use of reagents must also be minimized (especially if reagents require specific measures of disposal).

Regarding the huge sector of medical diagnostics (with a market volume of over $\$ 30$ billion), the state-of-the-art dictates that, a reliable patient profile requires a very large number of tests, each one dedicated to the detection of an individual target compound or pathogen. Due to the current availability of multiphasing screening machines, which can perform many blood tests quickly using very low sample volumes, there are more than 250 different blood tests and more than 60 urine tests currently available. Although the incidence of disease, in several cases, can be attributed to individual factors, this is by no means common in the case of complex and/or chronic ailments, such as cancer, neurodegenerative conditions and various forms of inflammatory disease. Having this in mind, researchers have focused their effort both on the development of biosensors applicable for multianalyte detection as well as on creating systems able to provide as much information as possible during a single assay, with as little time delay as possible (preferably monitoring at real-time conditions). One way to achieve this is by using cells as the biorecognition elements of choice.

Essentially every reported cell-based sensor system resides on the measurement of the interaction between cells and their environment. The cellular response to different analytes can vary in magnitude and duration, depending on the cell type and the nature of the cell-affecting compound or physicochemical condition. Nevertheless cells have frequently demonstrated themselves as quite sensitive detectors against a multitude of different target molecules. This trait, which has emerged as a result of relentless trial-and-error testing through countless millennia, has been exploited, on a regular basis, for the purpose of developing cellular biosensor principles with the promise of increased sensitivity and the ability of providing bioactivity-related information (which is generally not possible with conventional analytical systems, including older biosensor generations) [1]. The most prominent use of cell-based detection systems is toxicity testing, covering an impressive range of environmental challengers, such as heavy metals, complex organic molecules, endocrine disruptors and pesticide residues, to name a few.

Contrary to physiological and biochemical events on the singlecell level, coordinated responses within a given cell population are associated with important biological processes, such as cell division, tissue/organ development and conditioning to environmental stimuli. Examples of such coordinated responses include:

1. Bacterial quorum sensing: Bacteria can sense whether they have reached a critical density, by determining the concentration of an "autoinductor" molecule. In turn, they start forming biofilms, which are considerably more resilient to antibiotic treatments. The interference with these signaling systems, also known as quorum quenching, represents a promising strategy to tackle bacterial infections [2].

2. Tumor metastasis: Where cell-to-cell contact is one of the major factors affecting the migration of cancer cells and their metastatic potential. Part of the current oncology research is focused on identifying cell-to-cell adhesion molecules (e.g. membrane-bound proteins) as markers for indicating local and distant metastasis [3].

3. Neurophysiology, memory and consciousness: For example, neurons can be conditioned to respond to certain neurotransmitters so that repeated stimuli can lead to selected strengthening of synaptic contacts [4].

From the application point of view, improvements in understanding the nature of these coordinated responses could lead to the development of novel, highly advanced diagnostic systems serving, for example, as a prognostic tool for various diseases as well as high throughput platforms for screening novel antimicrobial drugs.

Even more challenging will be the design of non-invasive biosensors, monitoring interactions at the level of whole tissue, organ or even organism by means of a remote sensing principle. Although such capabilities still belong to the sphere of wishful thinking (at least at the operational level), there is an increasingly accumulating amount of experimental evidence for the feasibility of developing an actual "bioscanner" that will require no actual contact with the cellular sample under investigation.

More specifically, intracellular communication may be facilitated by physical interactions other than of chemical or electrical nature. These "other" interactions are measured as variations of electromagnetic field emissions by the cells, essentially covering the whole range of the electromagnetic spectrum, from a few $\mathrm{Hz}$ to several (thousands) $\mathrm{THz}$, including the visible wavelengths [5]. Different cellular phenomena attribute to these effects, such as membrane-cytoskeleton interactions,

*Corresponding author: Spyridion Kintzios, Department of Agricultural Biotechnology, Agricultural University of Athens, Greece, E-mail: skin@aua.gr

Received March 21, 2012; Accepted March 22, 2012; Published March 25, 2012

Citation: Kintzios S (2012) A Quantum Leap in Diagnostic and Analytical Science: Next Generation Sensing Devices Targeted on Distant Cell-To-Cell Communication. J Biosens Bioelectron 3:e107. doi:10.4172/2155-6210.1000e107

Copyright: (C) 2012 Kintzios S. This is an open-access article distributed under the terms of the Creative Commons Attribution License, which permits unrestricted use, distribution, and reproduction in any medium, provided the original author and source are credited. 
cytoskeleton vibration, membrane ion-channels and ultra-weak photon ("biophoton") emission, also due to the production of free radical species by mitochondria. It is not only possible to measure such alternative forms of communication in order to profile the status of a biological system and interpret the underlying functional processes; but also we can use cells to do the detector's job. It has been documented that distant cells can communicate through non-chemical, nonelectrical signaling. In the majority of reported cases, such remote communication is realized after a cytotoxic event is stimulated in one cell (the inducer) and elicits physiological responses in a second cell (the detector) which is separated from the inducer so that no direct or indirect chemical communication is possible.

Several sub cellular components have been indicated as the source of electromagnetic activity, the exact measurement of which could be used as the working principle of the novel cell-based detectors. A few examples are given below:

1. Microtubules: They are the building blocks of the cytoskeleton, responsible for cell shape and intracellular organization. They can generate oscillating electric fields in the frequency region from $\mathrm{kHz}$ to $\mathrm{GHz}$. Changes in the cytoskeletal structure have been shown to be involved in distant cell-to-cell communication [6].

2. Reactive oxygen species, resulting from the oxidative metabolism in mitochondria as well as cell membrane-associated processes (such as neural cell membrane depolarization and $\mathrm{Ca}^{2+}$ ion efflux) have been linked with bioluminescence phenomena, mostly referred to as spontaneous ultra weak biophoton emission [7]. This is another term for coherent electromagnetic radiation, due to the fact that the distance between cells is smaller than the wavelength of the radiation they emit. The coherent states appear to be fundamental for biological systems since they enable optimization of organization, information quality, pattern recognition and regulation of biochemical and morphogenetic processes.

3. Cell membranes, in particular mechanisms regulating cell membrane potential, oscillating in acoustic frequency range. For example, sound waves in the frequency range of 6-38 $\mathrm{KHz}$ have been found to coordinate proliferation between neighboring bacteria [8]

In conclusion, scientific progress in the field of unconventional intercellular communication has laid the foundation for the possible development of biosensing devices either able to measure electromagnetic emissions from biological systems or containing cells as the biorecognition elements. It is tempting to visualize the commercialization, in the not-to-far future, of hand-held detectors containing an array of living cells, each one dedicated to the synchronization with respective cell types in the assayed system, e.g. a human body. Should such devices become available, they are bound to change diagnostic technology radically.

\section{References}

1. Kintzios SE (2007) Cell-based biosensors in clinical chemistry. Mini Rev Med Chem 7: 1019-1026.

2. Romero M, Acuna L, Otero A (2012) Patents on quorum quenching: interfering with bacterial communication as a strategy to fight infections. Recent Pat Biotechnol 6: 2-12

3. Htwe TT, Karim N, Wong J, Jahanfar S, Mansur MA (2010) Differential expression of galectin-3 in advancing thyroid cancer cells: a clue toward understanding tumour progression and metastasis. Singapore Med J 51: 856859

4. Bailey CH, Giustetto M, Huang YY, Hawkins RD, Kandel ER (2000) Is heterosynaptic modulation essential for stabilizing Hebbian plasticity and memory? Nat Rev Neurosci 1: 11-20.

5. Cifra M, Fields JZ, Farhadi A (2011) Electromagnetic cellular interactions. Prog Biophys Mol Biol 105: 223-246.

6. Farhadi A, Forsyth C, Banan A, Shaikh M, Engen P, et al. (2007) Evidence for non-chemical, non-electrical intercellular signaling in intestinal epithelial cells. Bioelectrochemistry 71: 142-148.

7. Bischof M (2003) Introduction to integrative biophysics. Integrative Biophysics Biophotonics. Kluwer Academic Publishers, Dordrecht, The Netherlands.

8. Matsuhashi M, Pankrushina AN, Takeuchi S, Ohshima H, Miyoi H, et al. (1998) Production of sound waves by bacterial cells and the response of bacterial cells to sound. J Gen Appl Microbiol 44: 49-55. 